(C) The Association for Computational Linguistics and Chinese Language Processing

\title{
Constructing a Temporal Relation Tagged Corpus of Chinese Based on Dependency Structure Analysis
}

\section{Yuchang CHENG*, Masayuki ASAHARA* and Yuji MATSUMOTO*}

\begin{abstract}
This paper describes an annotation guideline for a temporal relation-tagged corpus of Chinese. Our goal is construction of corpora to be used for a corpus-based analysis of temporal relations among events. Since annotating all combinations of events is inefficient, we examine the use of dependency structure to efficiently recognize temporal relations. We annotate a part of Treebank based on our guidelines. Then, we survey a small tagged data set to investigate the coverage of our method. While we find that use of dependency structure drastically reduces manual effort in constructing a tagged corpus with temporal relations, the coverage of the methods achieves about $63 \%$.
\end{abstract}

Keywords: Temporal Entities, Event Entities, Temporal Reasoning, Event Semantics, Dependency Structure

\section{Introduction}

Extracting temporal information in documents is a useful technique for many NLP applications such as question answering, text summarization, machine translation, and so on. Temporal information includes three elements: 1. temporal expressions, which describe time or period in the real or virtual world; 2. event or situation expressions that occur instantaneously or that last for a period of time; 3. temporal relations, which describe the ordering relation between an event expression and a temporal expression or between two event expressions. There is a great deal of research dealing with temporal expressions and event expressions. Extracting temporal expressions is a subtask of NER [IREX committee 1999] and is widely studied in many languages [Mani et al. 2006b]. Normalizing temporal expressions has been investigated in evaluation workshops [Chinchor 1997]. Event semantics has been investigated in linguistics and in AI fields [Bach 1986]. However, research on temporal relation extraction is still limited. Temporal relation extraction includes the following issues: identifying events, anchoring an event to the timeline, ordering events, and reasoning of

\footnotetext{
* Nara Institute of Science and Technology, 8916-5 Takayama, Ikoma, Nara 630-0192, Japan

E-mail: \{yuchan-c, masayu-a, matsu\}@is.naist.jp
} 
contextually underspecified temporal expressions. To extract temporal relations, several knowledge sources are necessary, such as tense and aspect of verbs, temporal adverbs, and world knowledge [Mani et al. 2006b].

The goal of our research is to efficiently construct a temporal relation tagged corpus of Chinese. In English, TimeBank [Pustejovsky et al. 2006], a temporal information tagged corpus, is available for introducing machine learning approaches to automatically extract temporal relations. In Chinese, there is some related research on temporal expression extraction [Li et al. 2005]. However, there is no publicly available resource for temporal information processing in Chinese. Currently, such resources, event and temporal relation tagged corpora, are being made. Annotating all temporal relations of event pairs is time-consuming. Therefore, we propose a dependency structure based method to annotate temporal relations manually on a limited set of event pairs and extend the relations using inference rules. This method reduces manual effort. The dependency structure helps to detect subordinate and coordinate structures in sentences. We also describe a guideline for corpus annotation. Our annotation guideline is based on TimeML [Saurí et al. 2005], which was originally designed for English texts. We have developed a machine learning based dependency analyzer for Chinese [Cheng 2005] ${ }^{1}$ and a Chinese morphological analyzer [GOH 2006]. We can use our dependency analyzer to analyze raw text then use the output to annotate the temporal relations. However, in this paper, we use a syntactic tagged Chinese treebank (Penn Chinese Treebank) [Palmer et al. 2005] to create a temporal information annotated corpus. Finally, we survey the distribution of the temporal relations in our tagged corpus. We also evaluate the coverage of limited event pairs in our criteria.

\section{Background}

We investigated the distribution of events and temporal expressions in TimeBank [Pustejovsky et al. 2006] and Penn Chinese Treebank. TimeBank is a temporal information tagged corpus of English with TimeML annotation guideline. We found that the distribution of events and temporal expressions is uneven. Therefore, our corpus does not focus on the relations between an event and a temporal expression, but between two events. TimeML is a corpus annotation guideline of temporal information for English news articles. In this section, first, we introduce the resource -- TimeBank and describe the temporal relation links of original TimeML. Second, we investigate the distribution of events and temporal expressions in TimeBank and Penn Chinese Treebank. Finally, we analyze the temporal relation links to observe the correlation between dependency structure and TimeML links.

\footnotetext{
1 The dependency analyzer is trained on Penn Chinese Treebank. The accuracy of the head-modifier relation analysis is $89 \%$.
} 


\section{Dependency Structure Analysis}

Table 1. Tags of TimeML annotation

\begin{tabular}{|c|c|}
\hline Tags & Definition \\
\hline \multicolumn{2}{|r|}{ TimeML tags } \\
\hline EVENT & $\begin{array}{l}\text { Situations that "happen” or “occur”, includes tensed /untensed verbs, } \\
\text { nominalizations, adjectives, predicative clauses or prepositional phrases }\end{array}$ \\
\hline TIMEX3 & Temporal expressions, includes date, time and duration. \\
\hline SIGNAL & $\begin{array}{l}\text { Textual elements that make explicit the relation holding between two } \\
\text { entities }\end{array}$ \\
\hline MAKEINSTANCE & To create the actual realizations of an event \\
\hline \multicolumn{2}{|r|}{ Link tags } \\
\hline TLINK & $\begin{array}{l}\text { Temporal relation links, represents the temporal relationship holding } \\
\text { between two temporal entities (TIMEX3 and event) }\end{array}$ \\
\hline SLINK & $\begin{array}{l}\text { Subordinate links, represents contexts introducing relations between two } \\
\text { events }\end{array}$ \\
\hline ALINK & $\begin{array}{l}\text { Aspectual links, represents the relationship between an aspectual event } \\
\text { and its argument event }\end{array}$ \\
\hline
\end{tabular}

\subsection{Resources}

TimeML is a corpus guideline of temporal information for English news articles. Table 1 lists the definition of the tags. "EVENT", “TIMEX3" and "SIGNAL" tags in TimeML mark temporal entities such as event expressions and temporal expressions. Link tags annotate temporal relations between entities. The tag "TLINK" represents the temporal relationship between two entities. The definition of temporal relations using the tag "TLINK" is based on Allen's temporal relations [Allen 1983]. The tags "SLINK" and "ALINK" annotate the relations between a main event and its subordinate event. While the tag "ALINK" describes an aspectual relation, the tag "SLINK" describes a subordinate relation without explicit aspectual meaning. TimeBank is a temporal information tagged corpus that includes full temporal information (temporal expressions, events and temporal relations). The corpus is annotated according to the TimeML guidelines.

We create a temporal information tagged corpus for Chinese with our criteria because there is no such tagged corpus currently published. Our criteria include many elements of dependency structure. Sentences should be parsed to dependency structures; then, one should use the information of dependency structure to annotate temporal relations. Therefore, we used Penn Chinese Treebank [Palmer et al. 2005] as the original data and transfer phrase structures to dependency structures (See Section 4.1). 


\section{我昨天早上六点起床吃过早餐后搭公车上学}

(I waked up at 6 A.M. yesterday, ate breakfast and then go to school by bus.)

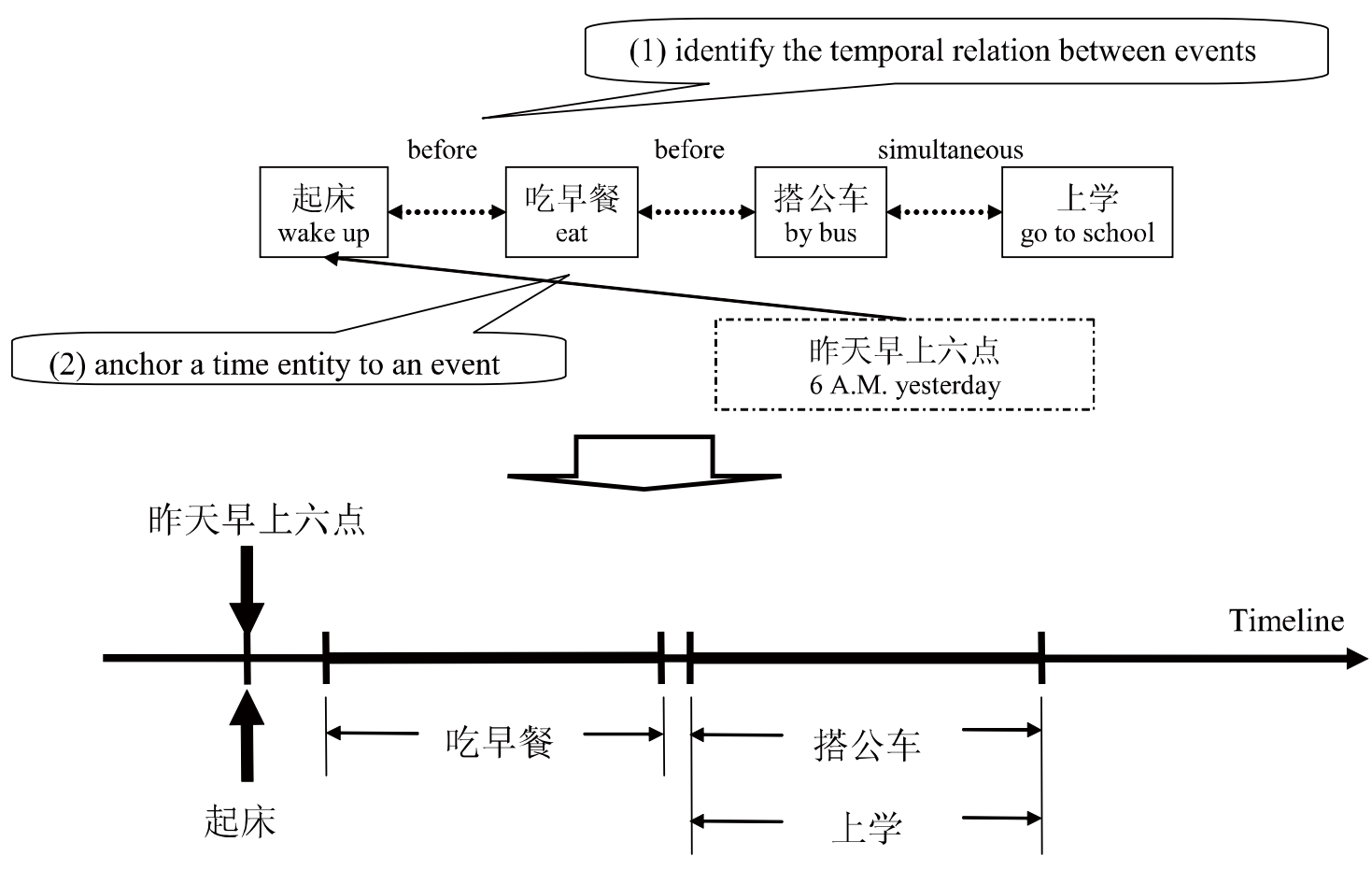

Figure 1. The temporal relations between events and a temporal expression

\subsection{The Temporal Relations: "between a temporal expression and an event" OR "between two events"}

Temporal relation includes anchoring a relation from an event to a temporal expression and ordering the relations between two events. Intuitively, ordering two events requires a temporal expression that can anchor events on the timeline. Previous research [Li et al. 2001] focused on simple anchoring problems, such as co-reference resolution between an event and a temporal expression. However, recent research ([Mani et al. 2006b], [Li et al. 2004]) has covered temporal relations depending on not only the temporal expressions but also world knowledge such as verb ontology in VerbOcean [Chklovski and Pantel 2004] and inference rules.

However, some events cannot be anchored on a timeline without ordering the events independently. For example, in Figure 1, there is one temporal expression “昨天早上六点” (6 A.M. yesterday) and four verb phrases (“起床” (wake up), “吃早餐” (eat breakfast), “搭公车” 


\section{Dependency Structure Analysis}

(by bus), and “上学” (go to school)) in the example sentence. For ordering these events (verbal phrases) on the timeline, we can analyze the temporal relationship between an event and a temporal expression. In this example, there is only one temporal expression “昨天早上 六点” (6 A.M. yesterday) that can be analyzed, and it is the anchor time of the event “起床” (wake up). Figure 1 (1) describes the temporal relations of adjacent event pairs ${ }^{2}$, and we can recognize some temporal relations by considering the event pairs rather than the temporal expression. These temporal relations are: the event “起床” (wake up) occurs before the event “吃早餐” (eat breakfast); the event “吃早餐” (eat breakfast) occurs before the event “搭公车” (by bus); and the events “搭公车” (by bus) and “上学” (go to school) occur at the same time. Figure 1 (2) describes the temporal relation between the anchor time “昨天早上六点” (6 A.M. yesterday) and the event “起床” (wake up). This temporal relation can anchor the event on the timeline. Combining the temporal relations in Figure 1 (1) and (2), the reader can recognize what happened and when they happened. Therefore, we can divide the process of recognizing the temporal information in the sentence into two steps: (1) recognizing the temporal relation between two events; (2) anchoring the events on the timeline. In extreme cases, we can think that a reader can recognize the situation by only considering the temporal relation between events, even if the reader does not know the anchor time of the events. Therefore, we think that annotating the temporal relations between event pairs is an independent task in temporal information processing. In this research, we focus on annotating the relations between two events.

Table 2. Distribution of tags in TimeBank

\begin{tabular}{|c|c|c|c|c|c|}
\hline \multicolumn{6}{|c|}{ Distribution of temporal entities tags } \\
\hline Tags & EVENT & MAKEINSTANCE & TIMEX3 & SIGNAL \\
\hline Number & 7935 & \multicolumn{7}{c|}{7940} & 1414 & 688 \\
\hline \multicolumn{6}{|c|}{ Distribution of temporal links in adjacent and dependency structure viewpoints } \\
\hline \multirow{2}{*}{ Entities } & all links & $\begin{array}{c}\text { adjacent } \\
\text { relations }\end{array}$ & $\begin{array}{c}\text { head-modifier } \\
\text { relations }\end{array}$ & $\begin{array}{c}\text { adjacent and head-modifier } \\
\text { relations }\end{array}$ \\
\cline { 2 - 7 } & Timex3 and event & 6418 & 3467 & 1372 & 4458 \\
\hline SLINK & Event and event & 3314 & 1757 & 1186 & 2826 \\
\hline ALINK & Event and event & 2932 & 2129 & 2174 & 2833 \\
\hline
\end{tabular}

Considering the distribution of the events in TimeBank (see Table 1 and Table 2), the number of events is more than the number of temporal expressions (TIMEX3). Therefore, to order the events, many events should share a temporal expression or should be analyzed as the temporal relation of event pairs with no corresponding temporal expression. We observe

\footnotetext{
2 These temporal relations of the adjacent event pairs do not include all recognizable temporal relations.
} 
similar things in Chinese corpora. We calculate the distribution of the temporal phrases and verbs in the Penn Chinese Treebank [Palmer et al. 2005]. There are 72,245 verbs (the words with POS-tags VV, VA, VE, VC) and 10,129 temporal phrases (the phrases with the tag “TMP”) in this Treebank. Only a portion of the verbs in an article have their own temporal expression (phrases), other verbs do not have direct temporal expression to anchor the verbs on the timeline. If we consider the verbs as the events in Treebank, most of the temporal relations are not between a temporal expression and an event, but between two events. To analyze the temporal relations between events that do not have their own temporal expression is necessary for recognizing the temporal information in Chinese articles.

\subsection{Adjacent Links in TimeBank}

TimeBank $1.2^{3}$ contains 183 articles with over 61,000 non-punctuation tokens. We investigate the distribution of temporal tags as shown in Table 2. TimeBank includes 9,615 links (TLINK, SLINK, and ALINK), of which, 5,763 links are the relations between adjacent entity pairs ${ }^{4}$ (an adjacent pair means the focal event and its linearly preceding event). According to the distribution, if we are able to recognize adjacent relations correctly (at least $60 \%$ $(5,763 / 9,615)^{5}$ of temporal relations are recognized), we expect to acquire more temporal relations with an additional process, such as adaptation of inference rules that we will describe in Section 4.4. We refer to the links of adjacent relations as “adjacent links”. To recognize the adjacent links of events, we annotate adjacent event pairs.

Additionally, we find that about $40 \%(2,296 / 5,763)$ of the links in the adjacent links are SLINKs and ALINKs. The links with the tag "SLINK" mean subordinate relations between events (not from an event to a temporal expression). Subordinate relation is the relationship between a focus event and a main event that the focus event depends on. SLINKs do not include TLINKs. If we extract SLINKs first, extracting other TLINKs from the remaining temporal entities would become simple. This observation gives us the idea that recognition of SLINKs is an important task for annotating adjacent relations.

\footnotetext{
${ }^{3}$ http://www.ldc.upenn.edu/

${ }^{4}$ The tag "TLINK" includes the temporal relations between document creation time and other temporal entities in an article, and includes the temporal relations between two matrix verb events in different sentences.

${ }^{5}$ An adjacent link could simultaneously be a head-modifier link. Therefore the numbers of the column "adjacent links" and the column "head-modifier links" in Table 2 are not complementary. The column "all links" is not the sum of "adjacent links" and "head-modifier links". Further more, we only consider the dependency structure of "sentences". If the relation links in Timebank cross different sentences, the dependency structures cannot recognize these links. The remnants of SLINKS that are not head-modifier relations are the links crossing different sentences.
} 


\section{Dependency Structure Analysis}

\subsection{Links on the Dependency Structure in TimeBank}

Since the majority of adjacent links are subordinate relations, we cannot analyze the temporal relations between contiguous pairs of matrix verb events without analyzing the structure of the subordinate relations, namely dependency structure. To calculate the distribution of links in TimeBank using dependency structure, we parse the sentences in TimeBank into the dependency structure and estimate the number of head-modifier (governor-dependent) relations that are SLINK or ALINK. We use the POS-tagger "TnT" [Brants 2000] to tag the sentences and use the MST parser ${ }^{6}$ [McDonald et al. 2005] to parse sentences to dependency structures. The column of "Head-modifier links" in Table 2 shows the number of each type of link that is a head-modifier relation. Seventy-three (2,331/3197) percent of S/ALINKs (SLINK + ALINK) in TimeBank are of head-modifier relations. This shows that dependency structure can be used to extract most S/ALINKs in English articles. Note that an event pair in a head-modifier relation link can also be one in an adjacent relation link.

Previous researchers have shown that syntactic information is useful for temporal information extraction [Li et al. 2004]. We use dependency structure for annotating temporal relations. The reason is that dependency structures are simpler and more comprehensible than phrase structures. The dependency grammar is composed of asymmetric head-modifier relations between words. We focus on the relation of event pairs. Dependency structure can describe the semantic relations between events clearly. Subordinate relations can be identified by the dependency structure. Therefore, dependency structure analysis is very useful for annotating the temporal relation.

\section{Strategy of Chinese Temporal Information Annotation}

We propose an annotation guideline for developing a Chinese temporal relation tagged corpus. The guideline is based on TimeML. TimeBank includes all possible temporal relations between two entities and is annotated manually. However, to annotate full temporal information of a newswire text requires considerable human effort and cost. To reduce human effort, we introduce several constraints on the original TimeML. First, we limit the definition of events to verbs. Second, we focus on three types of event pairs in a complete graph according to dependency structure and use inference rules to extend relations.

\subsection{The Definition of the Events}

First, we limit the definition of events to verbs. According to the TimeML guideline for English, verbs, nominalizations, adjectives, predicative clauses, or prepositional phrases can be events. However, recognizing an instance whether nominalization represents an event or

\footnotetext{
${ }^{6}$ We train the MST parser using Penn Treebank.
} 
not is difficult in Chinese articles. For example, a nominalization “电话(telephone)” could mean a telephone machine in the example “我买了一具电话(I bought a telephone)”, or could mean a telephone call in the example “他在电话中说...(In the telephone call, he said...).” Similar to the example, the semantic role of most of the aforementioned non-finite entities (nominalizations and adjectives) is ambiguous in the morphological analysis and the meaning of an entity depends on the context. In other types of events (predicative clauses and prepositional phrases), recognizing these entities from a context needs chunking techniques. The phrases / clauses usually have the hierarchical structure of verbs. It will be complicated to recognize these event entities when we extract the events automatically. It is also difficult to recognize events from all of the aforementioned entities except for verbs. Therefore, to simplify the process of recognizing events, we only regard verbs as events in our research.

It should be noted that we do not limit the domain of verbs. In the related research [Li et al. 2001], the researchers manually created a dictionary which included the common verbs in Chinese financial news articles and recognized the event using the dictionary. However, our original data do not limit the domain of articles. We should consider all of the verbs in corpus to assure the multiplicity of our corpus.

\subsection{Three Types of Event Pairs}

Second, we focus on three types of event pairs in the complete graph. The first type is adjacent event pairs. The second and third types are the head-modifier event pairs and the sibling event pairs in dependency tree representation of a sentence. The first type (adjacent event pairs as seen in Section 2.3) and the other two types (head-modifier or sibling event pairs as seen in Section 2.4) are not exclusive. According to our investigation in TimeBank, subordinate event pairs are head-modifier relations and coordinate event pairs are sibling relations. Therefore, using dependency structure can extract subordinate relations and coordinate relations in a sentence.

The three types of pairs are shown in Figure 2. The example phrase “停止拨付财政债券 安排的资金并起诉 (To stop providing funds that were prepared by financial bond, and to prosecute...) in Figure 2 has four events: “停止 (stop)”, “拨付 (provide)”, “安排 (prepare)” and “起诉 (prosecute)”. The temporal relations of all possible event pairs are shown in the row "All possible temporal relations" of the table in Figure 2. For example, the temporal relation: \{安排,拨付,before\}, means that the event “安排(prepare)” occurs before the event “拨付(provide)”.

The adjacent pairs of these events are \{停止-拨付, 拨付-安排, 安排-起诉\}, and these relations are shown in the row "Temporal relations of adjacent event pairs". However, the relation of the adjacent event pair “安排-起诉” is not useful information for readers because the event “安排 (prepare)” is a subordinate event of the event “拨付 (provide)” and it 


\section{Dependency Structure Analysis}

describes a past event as a supplement of the event “拨付 (provide).” The temporal relation between events “停止 (stop)” and “起诉 (prosecute)” is more useful than the relation between events “安排 (prepare)” and “起诉 (prosecute)” because events “停止 (stop)” and “起诉 (prosecute)” are coordinate events.

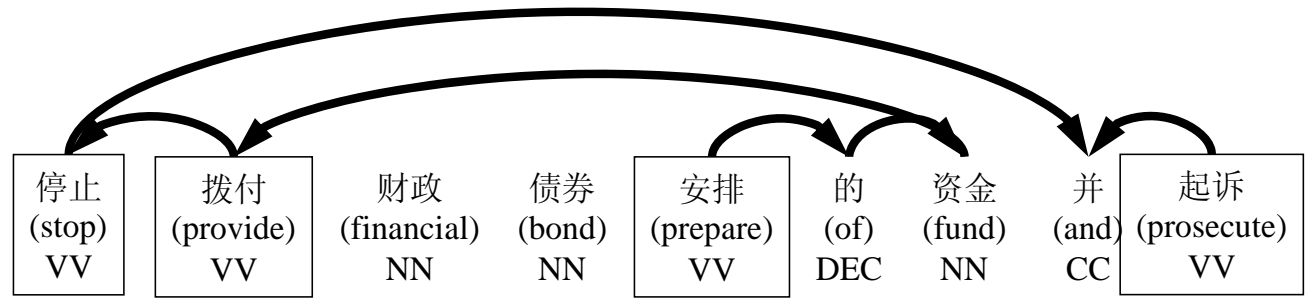

To stop providing funds that were prepared by financial bond, and to prosecute...)

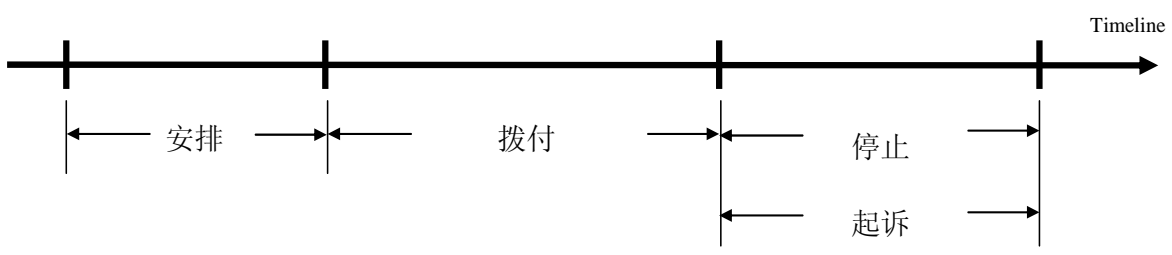

NOTE: a triple $\{A, B, C\}$ means that there is a relation $C$ between the focus event $A$ and related event $B$.

Adjacent event pairs:

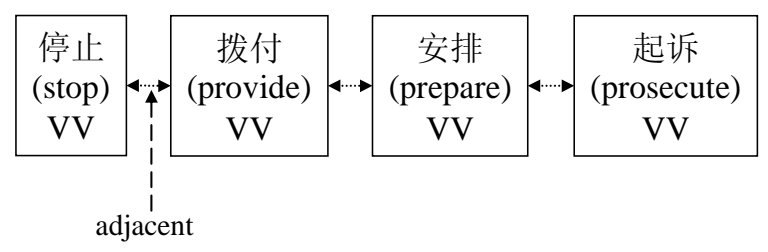

Dependency structure:

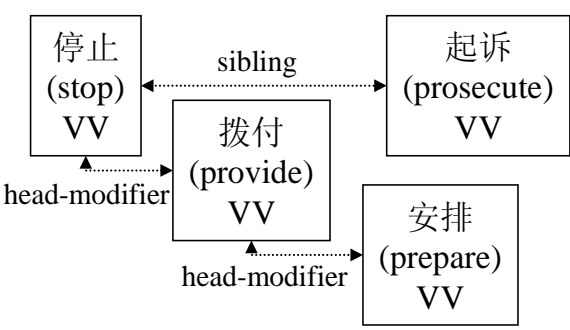

\begin{tabular}{|c|c|}
\hline Relation Types & Examples \\
\hline Temporal relations of adjacent event pairs & $\begin{array}{l}\text { \{安排,拨付,after }\} \text {, \{起诉,安排, after }\}, \\
\text { \{拨付,停止, before }\}\end{array}$ \\
\hline Temporal relations of head-modifier event pair & \{安排,拨付,after\}, \{拨付,停止, before $\}$ \\
\hline Temporal relations of sibling event pair & \{停止,起诉,simultaneous\} \\
\hline Extend event relations using inference rules & \{停止,安排,after\}, \{拨付,起诉,before\} \\
\hline True temporal relations & $\begin{array}{l}\text { \{安排,拔付,before }\},\{\text { 安排,停止,before }\}, \\
\text { \{安排,起诉,before }\},\{\text { 拨付,停止,before }\}, \\
\text { \{拨付,起诉,before }\},\{\text { 停止,起诉,simultaneous }\}\end{array}$ \\
\hline
\end{tabular}

Figure 2. The temporal relations in the example phrase 
In the example in Figure 2, a native annotator can recognize that the temporal relation between “安排 (prepare)” and “起诉 (prosecute)” is “before”. However, many event pairs like this example do not have an explicit temporal relation. To analyze this kind of event pairs (“安排 (prepare)” and “起诉 (prosecute)”), we should consider not only the adjacent observation of events but also dependency structure of sentences to acquire the correct temporal information. Moreover, if an adjacent event pair does not have understandable relation, the adjacent chain (adjacent links) will be segmented. The dependency structure can be used to connect the fragments of adjacent links.

The row "Temporal relations of Head-modifier event pairs" in the table shows the temporal relations of the head-modifier event pairs. We can determine these head-modifier event pairs as subordinate relations. For the event “起诉 (prosecute)”, the most important information is the relation between the coordinate event pair “停止 (stop)” and “起诉 (prosecute)". We define the event pairs that share a head event as a sibling event pair and show them in the row "Temporal relations of sibling event pairs" of the table. It should be noted that some adjacent event pairs are also head-modifier event pairs or sibling event pairs. The event pairs \{拨付-停止, 安排-拨付\} are adjacent event pairs and are head-modifier event pairs. Naturally, the event pair should have a similar temporal relationship from a different viewpoint.

\subsection{Use of Inference Rules}

After annotating these relation tags, we use inference rules (See Table 6), such as: "if event A occurs before event $B$, and event $B$ occurs before event $C$, then event $A$ occurs before event C”, to extend the temporal relations. The row "Extend event relations using inference rules" shows the temporal relations that are extended using inference rules. By annotating the three types of temporal relations and using the inference rules to extend the temporal relations, we do not need to annotate all possible event pairs, but we can acquire a number of useful temporal relations.

In Sections 2.3 and 2.4, we presented the concept that most of the temporal relations between events are among these three types in English. We expect that these three types of links (Adjacent event pairs, Head-modifier event pair, and Sibling event pair) in Chinese are more important than other links. In the next section, we describe our temporal information annotation guideline for Chinese. Section 5 shows the distribution of tags in our corpus and the coverage of the links induced by dependency structure.

\section{The Annotation Guideline}

This section describes our corpus annotation guideline. First, we introduce basic data and annotation tools. Second, we describe the definition of two temporal entities (events and 


\section{Dependency Structure Analysis}

signals). Third, we describe the concept of an event tree in an article with dependency structure. Fourth, we describe the attributes of the temporal entities and the temporal relation links. Finally, we compare our criteria and that of TimeML.

\subsection{Basic Data and Annotation Tools}

To recognize subordinate event pairs and head-modifier event pairs, we needed a dependency-parsed corpus. We used the Penn Chinese Treebank [Palmer et al. 2005] as the original data. Since the Penn Chinese Treebank does not include the head-modifier relations, we transformed phrase structures into dependency structures using head rules [Cheng 2005]. The head rules decide the head word of each phrase in the phrase structure, and then the phrase structure becomes a dependency tree. We annotate the temporal attributes and the temporal relations of events on a part of the Penn Chinese Treebank. Our corpus contains 151 Chinese news articles with 7,239 events and 49,691 tokens.

The punctuation “,” usually can be used in the semantic ending of a sentence in Chinese. To distinguish the meaning of the punctuation mark "," is difficult. We define the end mark of a sentence as the punctuation “。” (a period) in our corpus. The average length of sentences in the Penn Chinese Treebank is 27 words (507,222 words / 18,782 sentences) because a sentence in the Treebank could include several clauses which denote independent events.

We introduce the XML format for our data like TimeBank. We use an XML editor ${ }^{7}$ for annotating work. Figure 3 shows the window of the XML editor when we adopt it in our

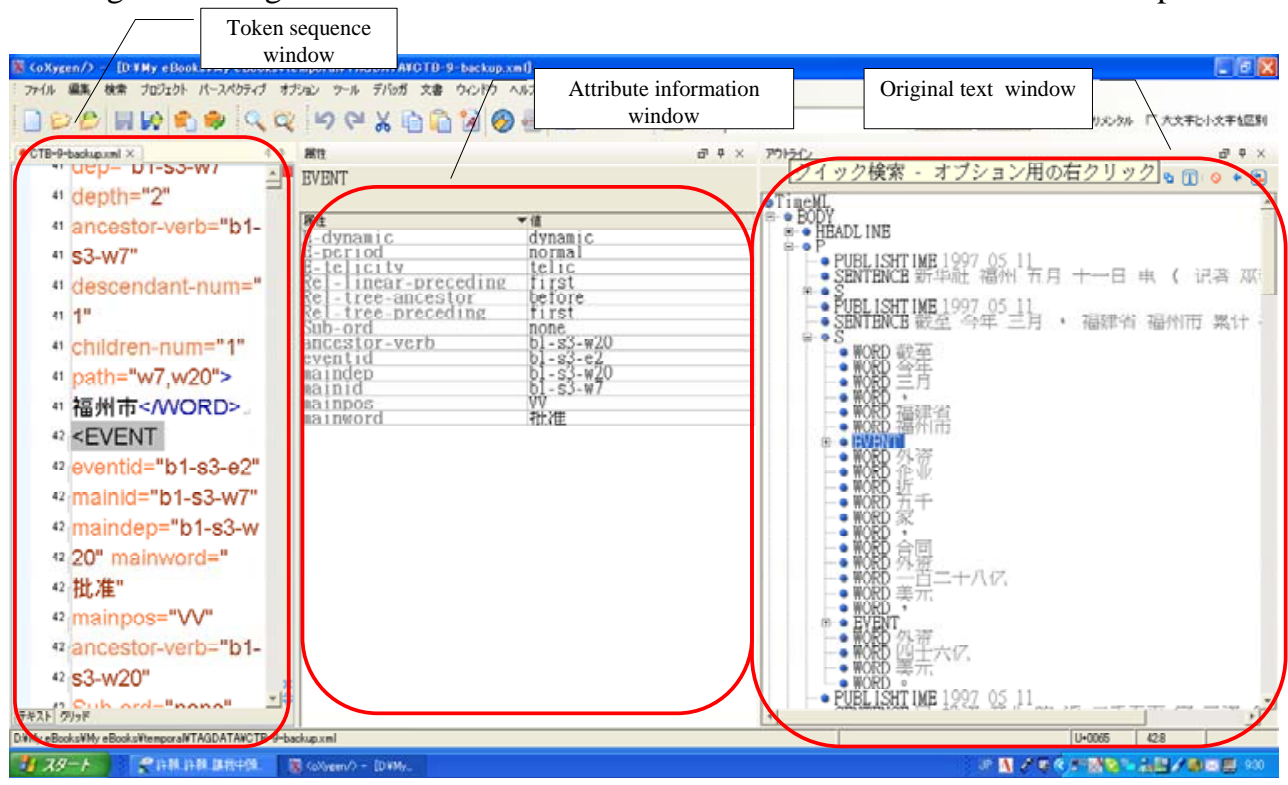

Figure 3. The working window for the annotator

\footnotetext{
7 We use the XML editor “<oXygen/>” for our work. (http://www.oxygenxml.com/)
} 
annotating work. It includes three sub-windows: "Token sequences window”, "Attribute information window" and "Original text window". The annotators refer the information in these windows and annotate the attributes of Attribute information windows.

\subsection{Definition of Events and Signals}

We annotate two sorts of entities; one is EVENTs, the other is SIGNALs. The definition of EVENT is based on the TimeML: an entity that describes a situation of happen, occur, state, or circumstance. However, we limit an event to one expressed by a verb in our guideline. According to the guidelines of Treebank, verbs serve as the predicate of a main clause or an embedded clause in corpus [Xia 2000]. We assume that a verb in a clause can be thought of as the representative entity of an event that the clause describes.

Verbs can be identified automatically, according to the POS tag of the word (the POS-tag: VV, VA, VC and VE). Most of the verbs in Treebank have the POS-tag "VV", which includes major verbs, such as raising predicates (“可能”(may be)), control verbs (“要”(want)), physical action (“飞”(fly)), psychological action (“讨厌”(hate)), and so on [Xia 2000]. The POS-tag “VA” is used for predicative adjectives, such as “齐全”(well-appointed). We consider predicative adjectives to be the same as an event because these predicative adjectives usually describe a statement. The predicative adjectives can modify a noun in another context, but in these cases the POS-tag of the predicative adjectives is an adjective "JJ". The difference can be analyzed in the step of morphological analysis or be distinguished in the original Treebank. Therefore, we also recognize predicative adjectives as a type of event according to the POS-tag. The POS-tag "VC" is the copula verb such as “是” (is). It describes a statement of a truth, such as the verb “是” (is) in the sentence: “我是学生” (I am a student), and we define these verbs as EVENT. The POS-tag "VE" describes the possessive or existential statement, such as the verb “有” (have) in the sentence “我有一本书” (I have a book). All these types of verbs are EVENTs and have the annotatable attribute in our criteria.

A SIGNAL is a textual element that makes explicit the relation between two temporal entities. In TimeML, it includes temporal prepositions, temporal conjunctions and prepositions signaling modality. Briefly, the original signals are composed by prepositions or conjunctions. A signal word could mean temporal or non-temporal relations depending on the contextual information. In the sentence “我昨天早上六点起床, 吃过早餐后搭公车上学”(I woke up at 6 A.M. yesterday, ate breakfast and then went to school by bus.) in Figure 1, the word “后” (after, then) is a signal word and describes the fact that the event “吃早餐”(eat breakfast) occurs before the event “搭公车” (by bus). However, the same word “后” (after, then) in the sentence “屋后有个花完” (There is a garden behind the house) means a location relation. Candidate words of SIGNAL in Chinese are limited. We collect these signal candidates according to the POS-tag standard of CKIP's corpus [CKIP 1993], which lists the SIGNAL 


\section{Dependency Structure Analysis}

candidates in the POS-tag “Ng” (Localizer). For example, the words “前, 后, 以上, 之前” are signal candidates. However, these signal candidates in Penn Chinese Treebank are not listed and are spread out in the prepositions ("P”), conjunctions (“CC”) and localizers ("LC”). To recognize the SIGNAL automatically, we use the SIGNAL candidate list that is collected from CKIP Treebank to annotate the words that correspond to the list as the "possible signal". A signal candidate word has an attribute with two classes: "time" and "non-time" to describe if it is a temporal signal word or not. We require the annotator to classify the signal candidate words manually. We will distinguish the use of signal words in different contexts using a machine learning classifier in future research.

\subsection{A Diagram of the Three Types of Event Pairs}

For annotating an article, we transform the parsed sentences into dependency structures. Figure 4 describes the relation of three types of event pairs in an article. There are two sentences with twelve events (from e1 to e12) in the figure, and the polygons with

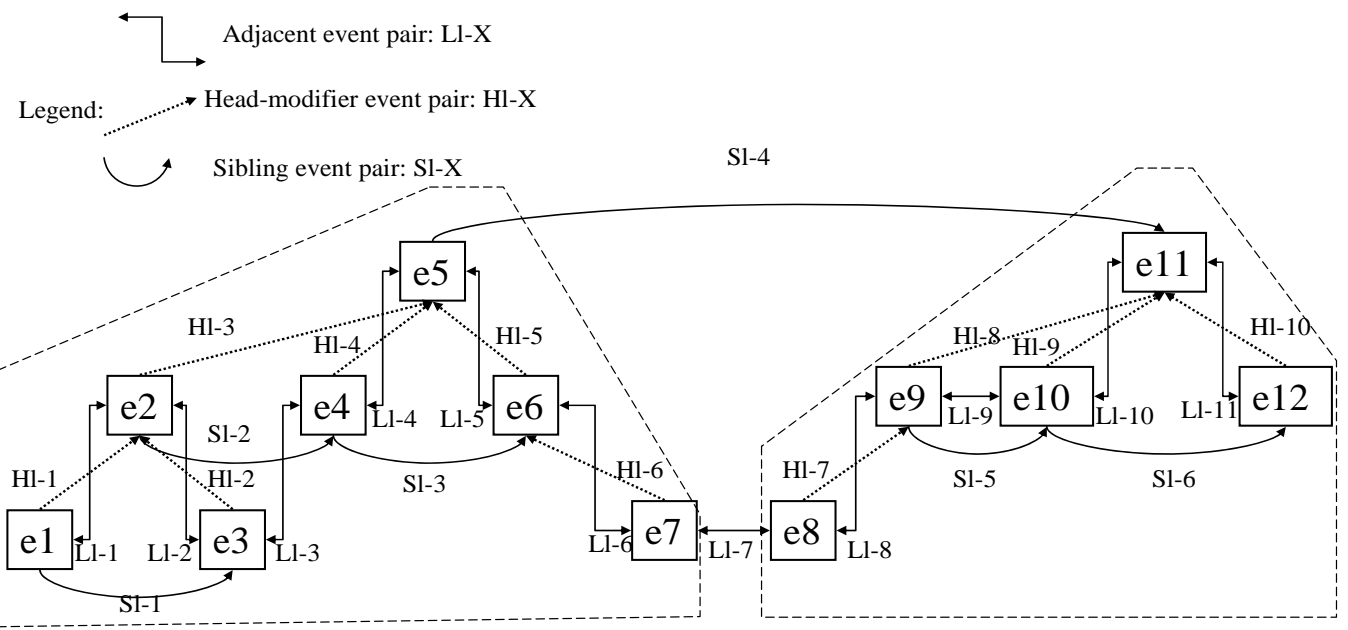

Sentence 1

Sentence 2

Figure 4. A diagram of the three types of event pairs and connecting the sentences

dashed-lines show the boundaries of sentences. The broken-line links show the adjacent event pairs (from Ll-1 to Ll-11). The dotted-line links show the head-modifier event pairs (from Hl-1 to Hl-10), and the curved links show the sibling event pairs (from Sl-1 to Sl-6). Some adjacent event pairs overlap head-modifier event pairs or sibling event pairs. Most of the three types of temporal relation links are in local structures (in sentence). To connect the temporal relations between sentences, the adjacent event pair links and the sibling event pair links can be used. The link Sl-4 and the link Ll-7 indicate the links that connect two sentences. The link "Sl-4" indicate the relations between the event "e5" and the event "e11". These events are the matrix events (main events) of "sentence 1" and "sentence 2". If we postulate that the article 
have a dummy root event and this dummy event is the parent event of all matrix events, the relation between the event " 5 " and the event "e 11 " is a sibling event pair. We can use the

\begin{tabular}{|c|c|}
\hline 属性 & 무주 \\
\hline \multicolumn{2}{|l|}{ WORD } \\
\hline 属性 & - 値 \\
\hline \multicolumn{2}{|l|}{ TMP } \\
\hline ancestor-verb & $b 1-s 3-w 7$ \\
\hline children-num & 1 \\
\hline dep & $b 1-s 3-w 7$ \\
\hline depth & 2 \\
\hline descendant-num & 1 \\
\hline path & $w 7, w 20$ \\
\hline pos & $\mathrm{NR}$ \\
\hline \multicolumn{2}{|l|}{ signal } \\
\hline \multicolumn{2}{|l|}{ verb-class } \\
\hline wid & $b 1-s 3-w 5$ \\
\hline word & 福州市 \\
\hline
\end{tabular}

\begin{tabular}{|l|l|}
\hline 属性 & \\
\hline EVENT & \\
\hline & \multicolumn{1}{l|}{ 마 $\times$ 值 } \\
\hline 属性 & dynamic \\
\hline E-dynamic & normal \\
\hline E-period & telic \\
\hline E-telicity & \\
\hline Rel-linear-pr... & \\
\hline Rel-tree-ance... & other \\
\hline Rel-tree-prec... & sub-ord \\
\hline Sub-ord & explanation \\
\hline ancestor-verb & b2-s9-w35 \\
\hline eventid & b2-s9-e3 \\
\hline maindep & b2-s9-w15 \\
\hline mainid & b2-s9-w11 \\
\hline mainpos & W \\
\hline mainword & 鼓励 \\
\hline & \\
\hline
\end{tabular}

Figure 5. Attribute windows for annotators.

inference rules on the connecting relations (Sl-4 and Ll-7) to deduce the temporal relations that cross into adjacent sentences.

\subsection{The Attributes of the Entities}

We annotate the two types of temporal attributes of events: the properties (dynamic, period and telicity) and the relations for limited event pairs (adjacent event pairs, head-modifier event pairs, and sibling event pairs). Some information of words and events can be annotated automatically, such as the POS-tag, head word, the path to the root of the sentence, and so on. The annotator refers to the annotated information to decide the most appropriate attributes of the temporal lines and event propertie s of each event. Figure 5 shows the attribute windows.

The left side window in Figure 5 shows the morphological information and the dependency information of a word, and the definition of these attributes is described in Table 3. All of these attributes are analyzed automatically. The "word" tag and the "POS" tag are similar to the original Treebank. The "TMP" tag refers to the phrase tag “*-TMP" in the Treebank.

The "verb-class" is a concept class of verbs. The verbs in Penn Chinese Treebank include four POS-tags (VV, VA, VC and VE). To give more semantic information of verbs, we define four classes of verbs to describe dynamic concepts: "state”, “change”, “action” and "mental." The class "state" describes a statement or a static situation, such as “齐全”(well-appointed). Most of the verbs of this class are the verbs with the POS-tag "VA". The class "change"

8 The Treebank includes "NP-TMP” (nominal phrase), “PP-TMP” (prepositional phrase), "LCP-TMP” (Localization phrase) and others. 


\section{Dependency Structure Analysis}

describes the change of statement, such as “变成”(become). The class “mental” describes a psychological action or state, such as “认为” (think) and “讨厌” (hate). The last verb class is “action”. We manually classify 23,979 verbs [GOH 2006] into these four classes. We assume that the concept of verb is important information for recognizing temporal relations. The verbs “射击”(shoot) and “认为”(assume) are in different classes. The class of the former verb “射 击”(shoot) is “action” and it usually means a time-bounded action (short period or instantaneous). The class of the latter verb “认为”(assume) is “mental” and it usually means a mental statement with long continuance. It should be noted that the "verb-class" is not necessarily the event class. The property of event could change with context. Actually, we require a lexicon of event semantics, such as Lexical Conceptual Structure [Jackendoff 1992], to classify the verbs in our dictionary. However, there is no Chinese lexicon with event semantic information that covers the verbs in our dictionary. Therefore, we classify the verbs into the four classes manually before we annotate the corpus. The temporal relation annotators are not required to classify the verbs when they annotate the corpus.

Table 3. Attributes of a word

\begin{tabular}{|c|c|}
\hline Attribute & Definition \\
\hline \multicolumn{2}{|r|}{ The dependency information } \\
\hline ancestor-verb & The ancestor verb of the focus word \\
\hline children-num & The number of children of the focus word \\
\hline Dep & The head word ID of the focus word \\
\hline Depth & The depth of the focus word in the dependency tree \\
\hline descendant & The number of descendant of the focus word \\
\hline path & The path from the focus word to the root of the dependency tree \\
\hline \multicolumn{2}{|r|}{ The morphological information } \\
\hline TMP & Is the focus word a part of a temporal expression? (yes or no) \\
\hline pos & POS tag \\
\hline signal & Is the focus word a signal word? (yes or no) \\
\hline verb-class & $\begin{array}{l}\text { The temporal meaning class of the verb ("state”, “change”, } \\
\text { "action" and "mental”) }\end{array}$ \\
\hline wid & The ID of the focus word \\
\hline word & The focus word \\
\hline
\end{tabular}

The right side window in Figure 5 shows the attributes of the focus event. Table 4 describes the attributes of an event. The attributes of an event include three parts: the information of the main verb in this event, properties of the event (E-dynamic, E-period, and E-telicity), and the temporal relations (Rel-liner-preceding, Rel-tree-preceding, Rel-tree-ancestor and Sub-ord). Properties of an event are the temporal characteristics of the 
event; these are different from the concept class of verbs that have already been described. These characteristics roughly correspond to the classification of verbs in Dorr and Olsen [1997]. These properties can describe the verb classification by Vendler [1967] or other classification through the combination of binary values. It includes telicity, dynamic characteristic, and occurrence period of a verb. The value of the tag "E-period" has a special value "forever" and the value "forever" describes an eternal action or situation. For example, the verb “绕” (circles) in the sentence “地球绕太阳” (the earth circles the sun) has the value "forever". Although some thesauri contain part of these properties of verbs, they are not publicly available. Additionally, as the properties could change in different contexts; we cannot annotate the properties automatically. We asked annotators not to classify events into several verb classes directly but instead select three binary attributes (E-dynamic, E-period, and E-telicity).

Table 4. Attributes of an event

\begin{tabular}{|c|c|c|}
\hline Attribute & values & Definition \\
\hline \multicolumn{3}{|c|}{ information of the main verb } \\
\hline ancestor-verb & & $\begin{array}{l}\text { The ancestor verb of the main verb of } \\
\text { the event }\end{array}$ \\
\hline eventid & & The ID of the event \\
\hline maindep & & The head word ID of the focus word \\
\hline mainid & & The ID of the main verb \\
\hline mainpos & & The POS tag of the main verb \\
\hline mainword & & The main verb \\
\hline \multicolumn{3}{|c|}{ the temporal properties of the event } \\
\hline E-dynamic & state, dynamic & Activity of event \\
\hline E-period & durative, instantaneous, forever & Period of event \\
\hline E-telicity & telic, non-telic & Telicity of event \\
\hline \multicolumn{3}{|c|}{ the temporal relation tag of the event } \\
\hline Rel-linear-preceding & Relations in Figure 6 & $\begin{array}{l}\text { Relation between the focus event and } \\
\text { the linear adjacent preceding event }\end{array}$ \\
\hline Rel-tree-preceding & Relations in Figure 6 & $\begin{array}{l}\text { Relation between the focus event and } \\
\text { the sibling event }\end{array}$ \\
\hline Rel-tree-ancestor & Relations in Figure 6 & $\begin{array}{l}\text { Relation between the focus event and } \\
\text { the ancestor event }\end{array}$ \\
\hline Sub-ord & $\begin{array}{l}\text { modal, explanation, condition, } \\
\text { none, report }\end{array}$ & $\begin{array}{l}\text { Subordinate type between the focus } \\
\text { event and the ancestor event }\end{array}$ \\
\hline
\end{tabular}


Dependency Structure Analysis

The four attributes (Rel-linear-preceding, Rel-tree-preceding, Rel-tree-ancestor, and Sub-ord) are temporal relations with another event. We describe these attributes in the following section.

\begin{tabular}{|c|c|c|c|}
\hline Relation types & Our criterion & TimeML & Allen \\
\hline $\begin{array}{lll} & \text { EVENT 1 } \\
\text { EVENT 2 } & \end{array}$ & \multirow{2}{*}{ AFTER } & AFTER & after \\
\hline \begin{tabular}{|l|l|}
\multicolumn{1}{l|}{} & EVENT 1 \\
EVENT 2 &
\end{tabular} & & IAFTER & met-by \\
\hline 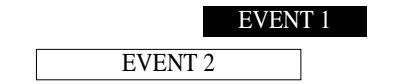 & \multirow{2}{*}{ OVERLAPPED-BY } & & overlapped-by \\
\hline $\begin{array}{l}\text { EVENT 1 } \\
\text { EVENT } 2 \\
\end{array}$ & & ENDS & finishes \\
\hline $\begin{array}{c}\text { EVENT 1 } \\
\text { EVENT } 2\end{array}$ & DURING & DURING/IS_INCLUDED & during \\
\hline $\begin{array}{l}\text { EVENT 1 } \\
\text { EVENT } 2 \\
\end{array}$ & BEGUN_BY & BEGUN_BY & started-by \\
\hline $\begin{array}{l}\text { EVENT } 1 \\
\text { EVENT } 2 \\
\end{array}$ & SIMULTANEOUS & SIMULTANEOUS/IDENTITY & equal \\
\hline \begin{tabular}{r|} 
EVENT 1 \\
EVENT 2 \\
\end{tabular} & INCLUDES & INCLUDES/DURING_INV & contains \\
\hline $\begin{array}{c}\text { EVENT 1 } \\
\text { EVENT 2 }\end{array}$ & ENDED_BY & ENDED_BY & finished-by \\
\hline $\begin{array}{l}\text { EVENT 1 } \\
\text { EVENT } 2\end{array}$ & \multirow{2}{*}{ OVERLAPS } & & overlaps \\
\hline \begin{tabular}{|r|} 
EVENT 1 \\
EVENT 2 \\
\end{tabular} & & BEGINS & starts \\
\hline \begin{tabular}{l|l} 
EVENT 1 & \\
\cline { 2 - 2 } & EVENT 2 \\
\end{tabular} & \multirow{2}{*}{ BEFORE } & IBEFORE & meets \\
\hline EVENT 1 & & BEFORE & before \\
\hline Non-tempoarl relation & first, ambiguous, none & & \\
\hline
\end{tabular}

Figure 6. Relation definitions among our critera, TimeML and Allen's work

\subsection{Annotating Links}

Our definition of temporal relations is based on TimeML language and Allen's research [Allen 1983]. The original definition of Allen's temporal relations is the relations between two time-intervals. We define four types of temporal relations between two events -Rel-linear-preceding, Rel-tree-preceding, Rel-tree-ancestor, and Sub-ord. The first three 
relations correspond to the relations that we described in Section 3 ("Rel-linear-preceding" refers to the adjacent event pairs, "Rel-tree-ancestor" refers to the head-modifier event pairs and "Rel-tree-preceding" refers to the sibling event pairs). The possible temporal relations are shown in Figure 6. EVENT 1 is the focal event and EVENT 2 is the related event. We group the temporal relation "overlapped-by" and "finished” in Allen's definition into the temporal relation "OVERLAPPED-BY" in our criteria because there are few instances of "overlapped-by" in our experience. We also group the temporal relation "overlaps" and "start" into the relation "OVERLAP”. The group “AFTER (BEFORE)” includes “after” and "met-by” ("before" and "meet”) in Allen's definition. This is because distinguishing "after" and "met-by" ("before" and "meet”) is difficult. Except for these groups, other relations are similar to TimeML and Allen's definitions. In addition to the recognizable temporal relations, we define three un-recognizable classes of relations: "first”, “ambiguous", and "none”. The value "first" means that the focus event does not have a comparable event. For example, the first event of a sentence does not have a preceding adjacent event; therefore, the value of the attribute "Rel-linear-preceding" is "first". The value "ambiguous" means that the temporal relation is suitable for more than one relation class. For example, if the event pairs could be annotated as "ENDED_BY" or "INCLUDES", the annotator should select the value "ambiguous". If the temporal relations cannot be decided (such as in an assumptive situation), the annotator is asked to select the value "none" for the focus event.

Table 5. Definition of subordinate class

\begin{tabular}{|l|l|}
\hline subordinate class & definition \\
\hline Modal & The focus event is introduced by the head event. \\
\hline explanation & The focus event explains the head event. \\
\hline condition & The focus event occurs if the head event is true. \\
\hline report & The head event is a "report" event. \\
\hline passive & The focus event is passive of the head event. \\
\hline possibility & The head event describes a possibility of focus event. \\
\hline
\end{tabular}

The last relation "Sub-ord" means the subordinate relation of a head-modifier event pair. We refer to TimeML in defining the subordinate relations. The annotator should annotate this relation without depending on the three temporal relations - "Rel-linear-preceding", "Rel-tree-preceding" and "Rel-tree-ancestor". Annotators can refer to the dependency structure of the focus event to recognize the subordinate event and its main event. The definition of the subordinate relations is described in Table 5. TimeML includes another link tag "ALINK" to annotate aspectual relations. We do not distinguish SLINK and ALINK and designate these two kinds of relations as the tag "Sub-ord". We assume that any subordinate relation could include a temporal relation. As the temporal relations include aspectual 


\section{Dependency Structure Analysis}

relations (such as BEGUN_BY and END_BY), the annotators can annotate the temporal relation between a sub-ordinate event and its head event to cover SLINK and ALINK.

Table 6. Inference rules

\begin{tabular}{|c|c|c|c|c|c|}
\hline & \multicolumn{5}{|c|}{ The relation between event B and event C } \\
\hline $\begin{array}{c}\text { The relation between } \\
\text { event A and event B }\end{array}$ & AFTER & BEFORE & DURING & INCLUDE & SIMULTANEOUS \\
\hline AFTER & AFTER & & & AFTER & AFTER \\
\hline BEFORE & & BEFORE & & BEFORE & BEFORE \\
\hline DURING & AFTER & BEFORE & DURING & & DURING \\
\hline INCLUDE & & & & INCLUDE & INCLUDE \\
\hline SIMULTANEOUS & AFTER & BEFORE & DURING & INCLUDE & SIMULTANEOUS \\
\hline & & The relation between event A and event C \\
\hline
\end{tabular}

Table 7. The attributes of the events in Figure 2

\begin{tabular}{|c|c|c|c|c|}
\hline event & $\begin{array}{c}\text { 停止 } \\
\text { (stop) }\end{array}$ & $\begin{array}{c}\text { 拨付 } \\
\text { (provide) }\end{array}$ & $\begin{array}{c}\text { 安排 } \\
\text { (prepare) }\end{array}$ & $\begin{array}{c}\text { 起诉 } \\
\text { (prosecute) }\end{array}$ \\
\hline \multicolumn{5}{|c|}{ the temporal properties of the event } \\
\hline E-dynamic & dynamic & Dynamic & dynamic & dynamic \\
\hline E-period & instantaneous & Durative & instantaneous & instantaneous \\
\hline E-telicity & telic & Telic & telic & telic \\
\hline \multicolumn{7}{|c|}{ the temporal relation tag of the event } \\
\hline Rel-linear-preceding & first & END_BY & BEFORE & AFTER \\
\hline Rel-tree-preceding & first & First & first & SIMULTANEOUS \\
\hline Rel-tree-ancestor & first & END_BY & BEFORE & first \\
\hline Sub-ord & none & Explanation & explanation & none \\
\hline
\end{tabular}

After we annotate the aforementioned temporal relations, we can use the inference rules to extend to more temporal relations. Table 6 shows the inference rules that we use in our experiment in Section 5.2. For example, if two temporal relations "Event A occurs during Event B" and "Event B occurs before Event C" are extracted, we can infer a new relation "Event A occurs before Event C". We will describe the inference rules in more detail in Section 5.2.

Table 7 describes the attributes of the events in the example sentence of Figure 2. In this example, the events “停止” (stop) and “起诉” (prosecute) are coordinate events, therefore the attribute "Rel-tree-preceding” of the event “起诉” (prosecute) is “SIMULATANEOUS”. The attributes "Rel-tree-preceding" of the other events are "first". The ancestor event of the events 
“拨付”(provide) and “安排”(prepare) is the same as their linear adjacent events, therefore the value of the tag "Rel-linear-preceding" is the same as the tag "Rel-tree-ancestor". Since the event “停止” (stop) is the first event and is the root event of the dependency tree, it does not have linear adjacent events nor ancestor events. Therefore, the values of tag "Rel-linear-preceding” and "Rel-tree-ancestor" are "first".

\subsection{Difference between our Guideline and TimeML}

Our corpus guidelines adopt many concepts and attribute values token from TimeML. However, our corpus criteria have several features that are different from TimeML. First, the goal of our research is to construct a machine learning based annotation system. All attributes can be annotated automatically after we complete a large corpus and train a machine learner. In TimeML, the annotators need to extract the temporal entities and relations using their knowledge, which requires a large amount of time. However, in our criteria, our annotators focus on the attributes of events and inference rules are used to extend temporal relations. We can create a large corpus according to our criteria, which would be more difficult using TimeML. Second, for recognizing the events of corpus automatically, we limit the events to the verbs, but TimeML includes more syntactic event constituents. To limit the event only to verbs can reduce the manual effort and preserve the major parts of all events.

When we use the temporal relation tagged corpus to train a machine learner, every attribute of our criteria can be trained. However, training the machine learner with a corpus tagged by the TimeML annotation scheme is more difficult than with our corpus. TimeML includes more difficult criteria. For example, the machine learner should identify the event phrase $^{9}$ (clause) in corpus. In a shared task of SemEval-2007 (Task 15: TempEval Temporal Relation Identification) [Verhagen 2007], participants use TimeBank as the corpus to identify the temporal relations in articles. However, they are not required to identify event phrases. Identifying event phrases requires chunking technology and world knowledge.

Finally, our criteria are based on dependency structure. TimeML does not consider any syntactic nor morphological information in their annotation. Our criteria are based on dependency structure and can describe the temporal relations of subordinate and coordinate event pairs clearly. In our experience, these criteria can provide annotators with useful information that help them to recognize the relations between events. Moreover, because verbs in Chinese do not have morphological change according to tense, recognizing the tense of an event needs the information of the modifier of the verb, such as a temporal expression "昨 天”(yesterday) or a temporal function word “已經”(have). This information directs the temporal relations analysis for Chinese. This information can be provided by dependency

\footnotetext{
9 The events in TimeML could be a noun phrase or verb phrase.
} 


\section{Dependency Structure Analysis}

structure, whereas TimeML does not emphasize this. Therefore, our criteria are more applicable than TimeML to the creation of a temporal relation tagged corpus of Chinese.

\section{The Corpus Distribution}

In this section, we report the distribution of the corpus annotation. We annotated a part of Penn Chinese Treebank and investigated the distribution of each attribute. Next, we investigated the coverage of the temporal relations using our proposed guideline.

\subsection{Distribution of Attributes in Tagged Corpus}

The Penn Chinese Treebank 5.0 contains 507,222 tokens, 18,782 sentences, and 890 articles. We will automatically analyze these attributes in the future. However, we need manually tagged training data to construct machine learning models. We use a part of the Penn Chinese Treebank (about 10\%) to construct a basic data set. As the consistency of the annotated corpus is not competent, we could not use it to get machine learning models before we repeated the annotating work to improve the consistency.

\section{Table 8. Distribution of the attributes}

\begin{tabular}{|l|l|l|}
\hline Attribute & Values & Number \\
\hline E-dynamic & State / dynamic & 5347 / 1892 \\
\hline E-period & durative / instantaneous / forever & 3024 / 4156 / 59 \\
\hline E-telicity & telic / non-telic & 3440 / 3799 \\
\hline Rel-linear-preceding & $\begin{array}{l}\text { (top four relations) } \\
\text { after / simultaneous / before / during }\end{array}$ & 2523 / 2065 /1091 / 463 \\
\hline Rel-tree-preceding & $\begin{array}{l}\text { (top four relations) } \\
\text { first / after / simultaneous / before }\end{array}$ & 5116 / 818 / 491 / 305 \\
\hline Rel-tree-ancestor & $\begin{array}{l}\text { (top four relations) } \\
\text { first / simultaneous / before / after }\end{array}$ & 1968 / 1816 / 1773 / 1073 \\
\hline Sub-ord & $\begin{array}{l}\text { (top four relations) } \\
\text { none / explanations / modal / report }\end{array}$ & 3622 / 1861 / 556 / 432 \\
\hline
\end{tabular}

The distribution of the attributes is summarized in Table 8. As the distribution of temporal relations is uneven and the space of this paper limited, we only show the top four types of temporal relations. Considering the tag "Rel-linear-preceding (adjacent event pairs)", the relation classes “AFTER / SIMULATANEOUS / BEFORE” are the most possible relations among the adjacent event pairs. Since we request the annotators to annotate as many temporal relations as possible, they used a considerable amount of world knowledge and contextual information in reading the articles. Therefore, the class "ambiguous" in tag "Rel-linear-preceding" is infrequent. The relation class "first" of the tag "Rel-tree-preceding (sibling event pairs)" means the focus event does not have any sibling event because events in 
similar sentences are structured in a hierarchical structure. There are few sentences that have events that modify the same event. Therefore, most events are singletons of their head events. In the tag "Rel-tree-ancestor (head-modifier event pairs)," the root event of the dependency structure does not have a head event and the correct selection of the tag "Rel-tree-ancestor" should be "first" in this case. In the tag "sub-ord (subordinate relation)", the value of the most meaningful types of subordinate relation are "explanations" and the majority of this attribute consists of the tag "none".

\subsection{The Coverage of the Links}

We investigate a small corpus to observe the performance of our criteria by comparing the results of our criteria and all possible event pairs. As described in Section 3, for $n$ events in an article, $\mathrm{nC} 2$ relations should be considered ${ }^{10}$. We can compare the relations of all pairs of events and the relations extracted by our criteria to observe the coverage of our criteria. However, it is difficult to annotate the temporal relations of all event pairs. For example, if an article contains 50 events, there are 1,225 event pairs $(50 \mathrm{C} 2)$. We cannot compare the two methods in a large corpus because the annotation cost is huge. Therefore, we select 50 articles in Penn Chinese Treebank and only use the first two paragraphs of each article to make our survey data. The small corpus includes 732 events (verbs) and 5,010 tokens.

We annotate the small corpus manually both by annotating all event pairs and by using our criteria. After annotation by our criteria, we use the inference rules shown in Table 6 to extend the relations. In previous research [Mani et al. 2006b], the inference rules could adopt some syntactic or semantic features ${ }^{11}$ of event pairs to extend more inference rules. To use syntactic/semantic features, experimental linguistic knowledge is needed to make an induction and we have not collected the linguistic knowledge yet. In this paper, therefore, we use the inference rules that only adopt unambiguous relations without syntactic/semantic features.

To observe the coverage of different methods, we survey four methods of extracting temporal relations. They are: 1 . Using the relationships of the adjacent event pairs (RLP is an abbreviation of Rel-linear-preceding), the head-modifier event pairs (RTA is an abbreviation

\footnotetext{
${ }^{10}$ We assume that the inverted relation pairs, such as "event A occurs before event B" and "event B occurs after event A", are different, because the combination $\mathrm{nC}_{2}$ only calculates a single direction of temporal relations, for example, the relation that forms event A to event B is extracted, but the relation that from event B to Event A is not considered. However, our method would extract two directions of temporal relation (the relation that from Event $\mathrm{C}$ to Event $\mathrm{A}$ is possible). If a relation between a combination event pair is extracted, we extend the inverse relation automatically (if event A occurs before event B, then event B occurs after event A).

${ }^{11}$ Such as the "POS" tag and the "TENSE" tag are used for creating inference rules in [McDonald et al. 2005].
} 


\section{Dependency Structure Analysis}

of Rel-tree-ancestor), and the sibling event pairs (RTP is an abbreviation of Rel-tree-preceding), then extending the relations by the inference rules (The column "RLP+RTA+RTP" in Table 9). 2. Only using the relations of the adjacent event pairs with the inference rules (The column "RLP" in Table 9). 3. Using the relations of the head-modifier event pairs and the sibling event pairs with the inference rules (The column "RTA+RTP" in Table 9). 4. Using three kinds of event pairs without the inference rules (The column "RLP+RTA+RTP w/o inference rules" in Table 9). For experimental convenience, we reduce the nine classes of temporal relations to five classes. The classes AAFTER, OVERLAP_BY, BEGUN_BY\} are reduced to the class “AFTER” and the classes \{BEFORE, OVERLAP, ENDED_BY $\}$ are reduced to the class "BEFORE." According to our annotator's experience, these subclasses are ambiguous in many event pairs; therefore, we group the classes to reduce the ambiguity.

Table 9. Results of the coverage evaluation

\begin{tabular}{|c|c|c|c|c|}
\hline & $\begin{array}{c}\text { RLP+RTA } \\
\text { +RTP }\end{array}$ & RLP & RTA+RTP & $\begin{array}{c}\text { RLP+RTA+RTP } \\
\text { w/o inference rules }\end{array}$ \\
\hline $\begin{array}{c}\text { Relations of Adjacent event pair } \\
\text { (The attribute Rel-linear-preveding-RLP) }\end{array}$ & 702 & 702 & 0 & 702 \\
\hline $\begin{array}{c}\text { Relations of Head-modifier event pair } \\
\text { (The attribute Rel-tree-ancestor-RTA) }\end{array}$ & 530 & 0 & 530 & 530 \\
\hline $\begin{array}{c}\text { Relations of Sibling event pair } \\
\text { (The attribute Rel-tree-preveding-RTP) }\end{array}$ & 205 & 0 & 205 & 205 \\
\hline Total extracted event relations & 1018 & 702 & 735 & 1018 \\
\hline $\begin{array}{c}\text { Extend event relations by using inference } \\
\text { rules }\end{array}$ & 4166 & 2005 & 2871 & 1018 \\
\hline True event pairs & 6646 & 6646 & 6646 & 6646 \\
\hline Recall & 0.63 & 0.30 & 0.43 & 0.15 \\
\hline
\end{tabular}

Table 9 describes the coverage of our proposed methods. We regard the understandable relations of all event pairs as the gold standard (the row "True event pairs") and we compare the result of our method with the gold standard. The row "Recall” shows the coverage of each method. We do not show the accuracy of each column because the extended relations are all included in the "True event pairs" and the precision of each column is "100\%".

The last column shows the case of using our criteria to annotate temporal relations without using the inference rules. The row "Extend event pair relations using the inference rules" in this column indicates the total number of events that are annotated by our criteria. It should be noted that an adjacent event pair could be also a sibling event pair or a head-modifier event pair. For example, the event pair the event “安排 (prepare)” and the 
event “拨付 (provide)” is both an adjacent event pair and a head-modifier event pair. It will be calculated twice in the two types of event pairs. Therefore, the number of the relations that we extract by our criteria is not equal to the total number of the three kinds of relation types (RLP+RTA+RTP > Total event pairs).

Intuitively, the combination of events must include all relations that could be extracted. The relations that we extract by our criteria must be included in the gold standard. In Table 9, the row "Total extracted event relations" is included in the true event relation. However, in our preliminary investigation (this result is NOT included in Table 9); the annotator does not consider any syntactic structure or full context in annotating the event pairs and then the extracted event relations are not completely consistent to the true event relations. Because this testing data set was annotated by an annotator but not completed in one day, the annotator does not remember the viewpoint before when he annotates the same instance. The annotator annotated the event combination first and then annotated the three types of event pairs of our criteria. The intuitive reorganization of event relations could be inconsistent with the dependency structure. Therefore we re-annotated the testing data several times to confirm the consistency of the relation attributes. This observation indicates the difficulty of constructing a corpus consistently.

According to our results, the precision of using "RTA+RTP" with the inference rules is better than the precision of only using "RLP" with the inference rules. The hypothesis in Section 3 is confirmed in the result. The head-modifier event pairs can connect some fragment structure and can extract many important relations that the adjacent event pairs cannot extract. The recall row shows the coverage of our method. We use three types of event pairs and the inference rules and acquire $63 \%$ relations of the gold standard can be extracted. One reason is that we only consider the absolute inference rules. We can add more inference rules that consider other syntactic or semantic information of events to extend the relations.

\section{Conclusion and Future Direction}

This research focuses on an annotation guidelines for a temporal relation tagged corpus of Chinese. The guidelines are based on the TimeML language, but we also use dependency structure information to acquire more meaningful temporal relations and to reduce manual effort. We define events as those expressed by verbs and define three types of links for event pairs. These types (the adjacent event pairs, the head-modifier event pairs, and the sibling event pairs) include most meaningful information, and we extend these relations using the inference rules.

To annotate temporal relations of all combinations of events requires nC2 manual judges. Our criteria require at most $3 n$ times of annotation. While the dependency structure based attributes reduce manual annotation costs, the limited relations preserve the majority of the 


\section{Dependency Structure Analysis}

temporal relations. The average working time required for one article (with 80 events) is about 30 minutes in our annotation work. It is shorter than the annotating work of TimeBank [Pustejovsky et al. 2006], which is 45 minutes for one article. We survey the coverage of our method with a small corpus. The result shows that our method covers about $63 \%$ of temporal relations. We expect that extension by our inference rules enables one to extract more temporal relations.

In future research, we will use the machine learning method to annotate the temporal relations. We annotated the Penn Chinese Treebank ceaselessly by our criteria. Once we annotate enough data, we will train it and, thereby, should reduce the inconsistency of our data.

\section{References}

Allen, J. F., "Maintaining Knowledge about Temporal Intervals," Communications of the ACM (Association for Computing Machinery), 26(11), 1983, pp. 832-843.

Bach, E., "the algebra of events”, Linguistics and Philosophy 9, Swets \& Zeitlinger Publishers, London, 1986, pp. 5-16.

Brants, T., TnT - $\quad$ A $\quad$ Statistical Part-of-Speech Tagger, http://www.coli.uni-saarland.de/ thorsten/tnt/, 1998.

Cheng, Y., Chinese Deterministic Dependency Analyzer: Examining Effects of Chunking, Root node finder and Global Features, MD thesis, NAIST, 2005.

Chinchor, N., MUC-7 named entity task definition, http://www.itl.nist.gov/iaui/894.02/related_projects/muc/proceedings/muc_7_proceedin gs/overview.html, 1997.

Chklovski, T., and P. Pantel, "VerbOcean: Mining the Web for Fine-Grained Semantic Verb Relations," In Proceeding of 2004 Conference on Empirical Methods in Natural Language Processing, 2004, Barcelona, Spain, pp. 33-40.

CKIP, “中文詞類分析,” Technical report no. 93-05, Institute of Information Science Academia Sinica, Teipei, 1993.

Dorr, B. J., and M. B. Olsen, "Deriving Verbal and Compositional Lexical Aspect for NLP Application," In Proceeding of 35th Annual Meeting of the Association for Computational Linguistics, 1997, Madrid, Spain, pp. 151-158.

GOH, C.-L., Unknown Word Identification for Chinese Morphological Analysis, PhD thesis, NAIST, 2006.

IREX Committee, Named entity extraction task definition, http://nlp.cs.nyu.edu/irex/NE/df990214.txt, 1999.

Jackendoff, R., Semantic Structure, The MIT Press, Cambridge, 1992. 
Li, W., K.-F. Wong, and C. Yuan, “Application and Difficulty of Natural Language Processing in Chinese Temporal Information Extraction," In Proceeding of the Sixth Natural Language Processing Pacific Rim Symposium, 2001, Tokto, Japan, pp. 501-506.

Li, W., K.-F. Wong, G. Cao, and C. Yuan, “Applying Machine Learning to Chinese Temporal Relation Resolution," In Proceeding of 42nd Annual Meeting of the Association for Computational Linguistics, 2004, Barcelona, Spain, pp. 582-588.

Mani, I., J. Pustejovsky, and R. Gaizauskas (ed.), the language of time, Oxford University express, Oxford, 2006a.

Mani, I., M. Verhagen, B. Wellner, C. M. Lee, and J. Pustejovsky, "Machine Learning of Temporal Relations," In Proceeding of the joint conference of 21st International Committee on Computational Linguistics and 44th Annual Meeting of the Association for Computational Linguistics, 2006, Sydney, Australia, pp. 753-760.

McDonald, R., F. Pereira, K. Ribarov, and J. Hajic, "Non-Projective Dependency Parsing using Spanning Tree Algorithms," In Proceeding of 2005 Conference on Empirical Methods in Natural Language Processing, 2005, Vancouver, Canada, pp. 523-530.

Palmer, M., F.-D. Chiou, N. Xue, and T.-K. Lee, Chinese Treebank, version 5.1, http://www.ldc.upenn.edu/Catalog/CatalogEntry.jsp?catalogId=LDC2005T01U01, 2005.

Pustejovsky, J., M. Verhagen, R. Sauri, J. Littman, R. Gaizauskas, G. Katz, I. Mani, R. Knippen, and A. Setzer, TimeBank, version 1.2, http://www.ldc.upenn.edu/ Catalog/CatalogEntry.jsp?catalogId=LDC2006T08, 2006.

Saurí, R., J. Littman, B. Knippen, R. Gaizauskas, A. Setzer, and J. Pustejovsky, TimeML Annotation Guidelines, version 1.2.1, http://www.timeml.org/ site/publications/specs.html, 2005.

Vendler, Z., Verbs and Times, Linguistics in Philosophy, Cornell University Press, Ithaca, 1967.

Verhagen, M., R. Gaizauskas, F. Schilder, M. Hepple, G. Katz, and J. Pustejovsky, “SemEval-2007 Task 15: TempEval Temporal Relation Identification,” In Proceeding of $4^{\text {th }}$ International Workshop on Semantic Evaluations, 2007, Prague, Czech, pp. 75-80.

Wu, M., W. Li, Q. Lu, and B. Li, "CTEMP: A Chinese Temporal Parser for Extracting and Normalizing Temporal Information," In Proceeding of 2nd International Joint Conference on Natural Language Processing, 2005, Jeju Island, Korea, pp. 694-706.

Xia, F., The Part-Of-Speech Tagging Guidelines for the Penn Chinese Treebank, http://www.cis.upenn.edu/ chin, 2000. 